\title{
"Fou rire prodromique" heralding a brainstem stroke
}

\author{
G M Wali
}

\begin{abstract}
A patient manifested pathological laughter heralding the onset of brainstem stroke leading to a "locked-in" state. The pathological laughter did not recur. MRI revealed a bilateral ventral pontine infarct. The clinico-anatomical correlations of this rare phenomenon of fou rire prodromique are discussed.
\end{abstract}

$(\Im$ Neurol Neurosurg Psychiatry 1993;56:209-210)

Fou rire prodromique is a neurological event characterised by the occurrence of pathological laughter at the onset of stroke. It is rarely reported $^{1-3}$ and its neuroanatomical basis is unclear. The clinical and anatomical MRI correlates of the present case are discussed in the light of previous reports.

\section{Case report}

A 35 year old, previously healthy, woman developed transient left faciobrachial weakness which lasted about 15 minutes. Three days later she developed a mild throbbing headache which appeared in the evening and persisted until the next morning when she developed the neurological event. After a warm shower she suddenly began to laugh. The laughter was inappropriate to the situation and continued for nearly 15 minutes at the end of which it became a low grade giggle. This lasted for another 15 minutes and ended abruptly when she collapsed and became immobile due to gross weakness of the limbs. Her eyes remained open and she was unable to talk. Blinking was present. She remained conscious throughout and had no automatic movements or convulsions.

On admission, her heart rate was 120 beats

Department of Neurology, Jawaharlal Nehru Medical College, KLE Society's Hospital and Medical Research Centre, Nehru Nagar, Belgaum, Karnataka State, India G M Wali

Correspondence to: Dr Wali, Department of Neurology, KLE Society's Hospital and Medical Hespital and Medical Nagar, Belgaum 590010, Karnataka State, India

Received 18 March 1992. Accepted 12 May 1992 sugar, cholesterol, urea, creatinine and electrolytes performed soon after admission were normal. CT of the brain the next day revealed a hypoluscent area in the brainstem at the level of the pons. The chest radiograph, ECG and echocardiogram were normal. Serological tests for syphilis and collagen vascular disease were negative. A lumbar puncture CSF study was also normal.

MRI performed in the fifth week revealed a bilateral, asymmetrical infarct of the basis pontis. The tegmentum was preserved. In the sagittal view (fig 1) the prong-shaped lesion involved almost the middle three-fifths of the basis pontis. The axial view (fig 2) demonstrated that the lesion predominantly involved the left half of the basis pontis. The right half was only partially involved in its anterior third. No lesions were seen at other sites. An EEG showed bilateral, diffuse, mild slowing of the background activity in the range $7-8 \mathrm{~Hz}$. No epileptiform discharges were seen. An angiogram was refused.

The patient improved partially over a period of one month. She could flex her right arm and leg spontaneously. Vocalisation was restricted to groaning. Her mood remained relevant to the situation and she did not exhibit any episodes of pathological laughter or crying. She died of pneumonia after six weeks. Necropsy was not performed.

\section{Discussion}

This patient developed the "locked-in" syndrome as a result of bilateral infarction of the basis pontis, demonstrated by MRI. The shape and extent of the infarct suggest that it occurred through occlusion of the paramedian branches of the basilar artery at the level of pons. ${ }^{4}$ The temporal profile of the neurological symptoms suggests the possibility of a thrombotic event. As the tests for specific aetiologies, such as syphilis, tuberculosis and collagen vascular disease were negative, the possibility of atherosclerosis is considered although it is uncommon at this age. ${ }^{4}$

Laughter is termed pathological when it is inappropriate and continuous. It is not associated with expected mood changes and there is an absence of voluntary control over the extent and duration of the facial expressions. Spasmodic pathological laughter could occur as a release phenomenon following destructive lesions of the CNS as seen with pseudobulbar palsy, epileptic phenomenon (gelastic epilepsy) 
Figure 1 MRI sagittal view of the lesion $(T$, weighted image). The prong-shaped lesion involves the middle three-fifth of the basis pontis.

Figure 2 Axial view showing extensive involvement of the left half of the basis pontis, whereas the anterior right half is minimally involved.

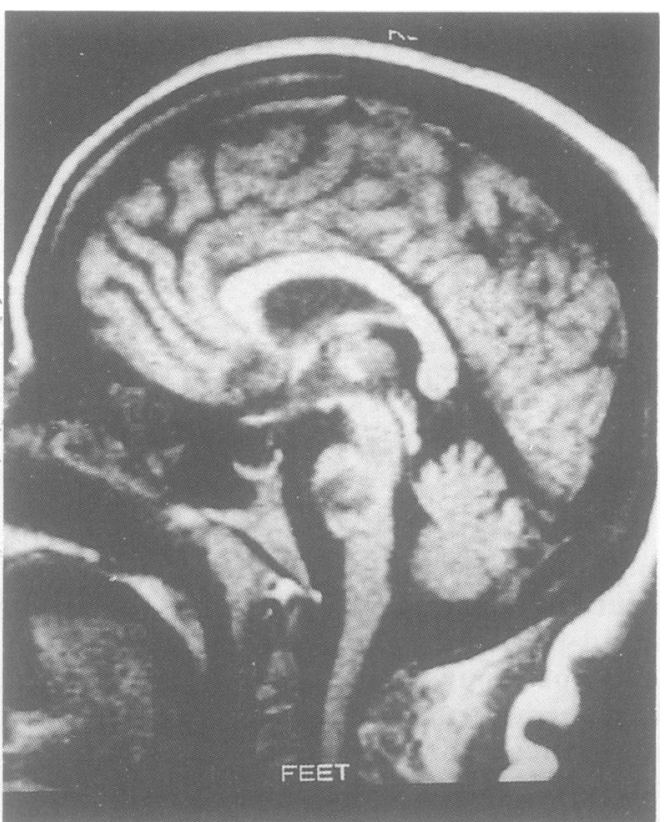

and as a manifestation of psychiatric disorders such as schizophrenia and hysteria. ${ }^{5}$ Commonly it is associated with pathological crying. In contrast, fou rire prodromique is a rare syndrome in which pathological laughter occurs at the onset of an apoplectic event. It was described for the first time by Fere in 1903. ${ }^{1}$ His patient was an elderly man who developed a left hemiplegia following a bout of laughter. Until his death 18 months later, the patient had no further attacks of pathological laughter. Poeck ${ }^{2}$ has cited the reports published by Badt (1927) and Andersen (1936) in the German literature. In both these cases there was haemorrhage into both thalami and internal capsules. The patient reported by Andersen suffered uncontrollable laughter which persisted until his death 24 hours later. Martin $^{3}$ reported a fatal case of ruptured basilar artery aneurysm in which death was preceded by an attack of pathological laughter. The large aneurysm which pressed upon the upper brainstem and posterior part of third

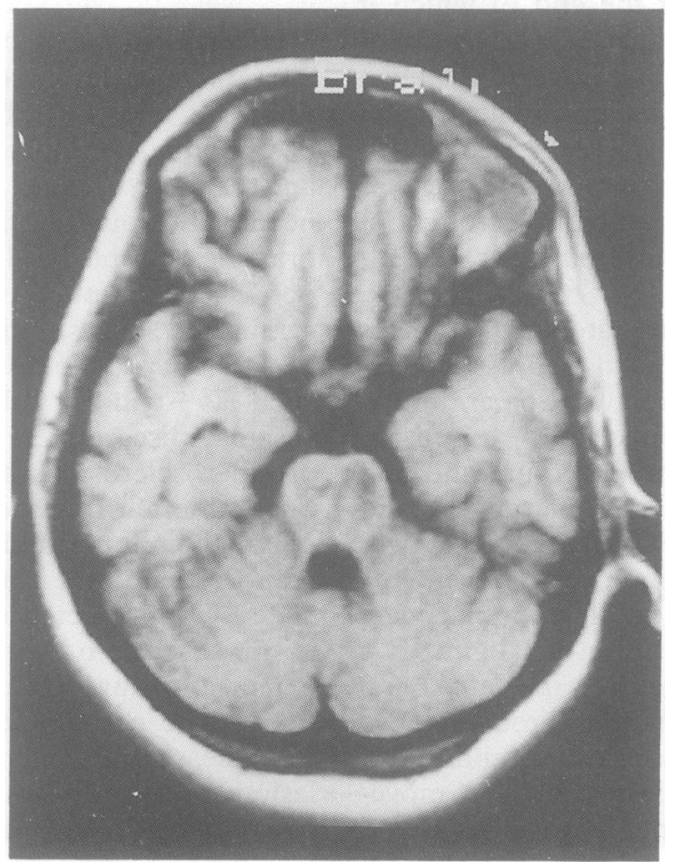

ventricle also destroyed the mammillary bodies. The present case belongs to this rare group of strokes. The pathological laughter preceded the onset of stroke and did not recur, indicating that it was not a part of the pseudobulbar syndrome. It was also not a gelastic seizure because there were no automatisms or altered sensorium. The EEG did not reveal any epileptiform discharges.

There is no clear idea about the lesions that can produce pathological laughter. Poeck and Pilleri $^{6}$ have analysed 30 cases that were verified at necropsy. The various sites of lesion demonstrated in these cases include the internal capsule with adjacent basal ganglia, substantia nigra, cerebral peduncles, caudal hypothalamus, bilateral foci in the thalamus and bilateral lesion of the pyramidal tract along with the extrapyramidal fibres accompanying them. Recently Asfora et $a l^{7}$ have reported an interesting case of angiographically occult pontine vascular malformation presenting solely with pathological laughing and crying. Their data suggest that pathological laughing and crying can occur without other features of pseudobulbar palsy.

The precise neuroanatomy of normal laughter is unknown. The most tenable suggestion was presented by Wilson. ${ }^{8} \mathrm{He}$ proposed the presence of a centre linking the facial nucleus in the pons with that of the tenth nerve in the medulla and the phrenic nuclei subserving respiratory movements. These connections are a requisite for the faciorespiratory coordination of laughter. $\mathrm{He}$ also postulated an integrative centre in the medial thalamus, hypothalamus and subthalamus. In turn, this centre is considered to be under the voluntary control of the corticobulbar tracts, and involuntary control of the respiratory tracts, which extend from the orbital gyrus of the frontal lobe and descend to the bulbar nuclei. These respiratory tracts have both accelerator and decelerator functions which are needed during laughter. Poeck ${ }^{2}$ has speculated that there is a tonic and phasic innervation of the movements of facial expression. In the fou rire prodromique there is a loss of the tonic control of laughter and, in spasmodic pathological laughter, there is a loss of the phasic control. Based on these proposals, it is possible that the patient's ventral pontine lesion led to the disruption of tonic innervation of the faciorespiratory centre in the lower brainstem.

1 Fere MC. Le fou rire prodromique. Rev Neurol 1903;11: 353-8.

2 Poeck K. Pathologic laughter and crying. In: Vinken PJ, Bruyn GW, Klawans HL (eds). Handbook of clinical neurology. Amsterdam: Elsevier, 1985:219-26.

3 Martin JP. Fits of laughter (sham mirth) in organic cerebral disease. Brain 1950;70:453-64

4 Awada A, Ammar A, Rajeh SA, Borollosi M. Excessive sweating: an uncommon sign of basilar artery occlusion. $\mathcal{F}$ Neurol Neurosurg Psychiatry 1991;54:277-8.

5 Black DW. Pathologic laughter - a review of the literature. $f$ Nerv Ment Dis 1982;170:67-71.

6 Poeck K, Pilleri G. Pathologisches laschen and Weinen. Schweiz Arch Neurol Psychiatr 1964;92:323-70

7 Asfora WT, DeSalles AAF, Abe M, Kjellberg RN. Is the syndrome of pathological laughing and crying a manifestation of pseudobulbar palsy? I Neurol Neurosurg Psychiatr 1989;52:523-5.

8 Wilson SAK. Some problems in neurology. II. Pathologic laughing and crying. I Neurol Psychopathol 1924;16: 299-333. 